EUROPEAN ORGANIZATION FOR NUCLEAR RESEARCH

CERN - SL DIVISION

CERN-SL-2000-051 BI

\title{
FIRST RESULTS OF THE BEAM GAS IONIZATION PROFILE MONITOR (BGIP) TESTED IN THE SPS RING
}

\author{
Arauzo A., Bovet C., Koopman J. and Variola A.
}

\begin{abstract}
The BGIP is a proposal for a new, non-destructive beam profile monitor for the future Large Hadron Collider (LHC). This device provides the rms beam size value by means of the analysis of the velocity spectrum of the rest gas ions created and accelerated by the beam itself. After a thorough computer simulation study of the related physics, a first prototype of the BGIP has been conceived, built up and installed in the SPS main ring during 1999. This paper contains a short presentation of the simulation work and a description of the test set-up. The first experimental results are presented and compared with theoretical computations.
\end{abstract}

Presented to the Beam Instrumentation Workshop in Boston - USA

8 - 11 May 2000

Geneva, Switzerland

July 2000 


\title{
First results of the Beam Gas Ionization Profile Monitor (BGIP) tested in the SPS ring
}

\author{
A. Arauzo, C. Bovet, J. Koopman, A. Variola \\ CERN-SL, CH-1211 Geneva 23, Switzerland
}

\begin{abstract}
The BGIP is a proposal for a new, non-destructive beam profile monitor for the future Large Hadron Collider (LHC). This device provides the rms beam size value by means of the analysis of the velocity spectrum of the rest gas ions created and accelerated by the beam itself. After a thorough computer simulation study of the related physics, a first prototype of the BGIP has been conceived, built up and installed in the SPS main ring during 1999. This paper contains a short presentation of the simulation work and a description of the test set-up. The first experimental results are presented and compared with theoretical computations.
\end{abstract}

\section{INTRODUCTION}

The BGIP monitor has been conceived for measuring the transversal beam size of the LHC beam over the whole energy range, from injection at $450 \mathrm{GeV}$ to collision at $7 \mathrm{TeV}$.

The Orsay group first developed this type of detector, for the FFTB at SLAC [1,2]. This paper describes the conception of this detector for being used with a proton beam and for short bunch to bunch spacing ( $25 \mathrm{~ns}$ ).

The principle of detection is based on the measurement of the total momentum kick given to ions by the space charge field of the beam. The positive ions are coming from the residual gas, which has been ionized by the beam itself. The acquired energy by the ions depends on the beam size, so by measuring the ion velocity we can determine the beam dimensions. With this purpose, ions from the beam vacuum chamber are collected through a slit and a dipole magnet is used as a spectrometer to allow the conversion of the distribution in velocities in a distribution of positions in a MCP.

\section{THEORETICAL BACKGROUND AND SIMULATIONS}

The rate at which the ions arrive at the detector depends on the ionization rate and the angular aperture of the collector slit. The ionization rate is directly proportional to the intensity of the beam and to the pressure of the residual gas. For LHC nominal conditions, assuming a slit $2 \mathrm{~cm}$ long and $0.2 \mathrm{~cm}$ wide, the counting rate is of the order of $10^{5}$ ions $\cdot \mathrm{s}^{-1}$ [3]. The most likely expected ions are $\mathrm{H}^{+}$and $\mathrm{H}_{2}{ }^{+}$.

The space charge field of the beam causes ion acceleration. The electric field expands in the transverse plane due to Lorenz contraction, and the transverse 
distribution of charge is assumed to be Gaussian, with rms values $\sigma_{\mathrm{x}}$ and $\sigma_{\mathrm{y}}$. The components of the transverse electrical field are calculated as a function of the transverse dimensions for a given longitudinal position in the bunch. In the particular case of a round beam, the field becomes radial.

The field provides an outward directed acceleration for the positive ions. The largest velocities are reached by the ions created near the beam center. Ions receive kicks from several bunches in their drift to the vacuum chamber wall.

\section{Simulations for the LHC beam}

The LHC beam can be considered as a round beam, with a transversal rms size, $\sigma_{\mathrm{r}}$. The electrical field is radial and is given by:

$$
E_{r}=2 N_{p} \frac{r_{e} m_{e} c^{2}}{e} \frac{1-e^{-r^{2} / 2 \sigma_{r}^{2}}}{r}\left(\frac{1}{\sigma_{z}} e^{-z^{2} / 2 \sigma_{z}^{2}}\right)
$$

where $\mathrm{N}_{\mathrm{p}}$ is the number of protons per bunch.

An example of the ion speed distribution obtained in the slit is given in Fig. 1. A sharp cut off can be observed for the maximum velocity values. By applying a linear fit to this edge, a prediction for the maximum velocity for the ions, $v_{f m}$, is obtained. A logarithmic dependence has been found for $\mathrm{v}_{\mathrm{fm}}{ }^{2}$ with the beam dimension $\sigma_{\mathrm{r}}$.

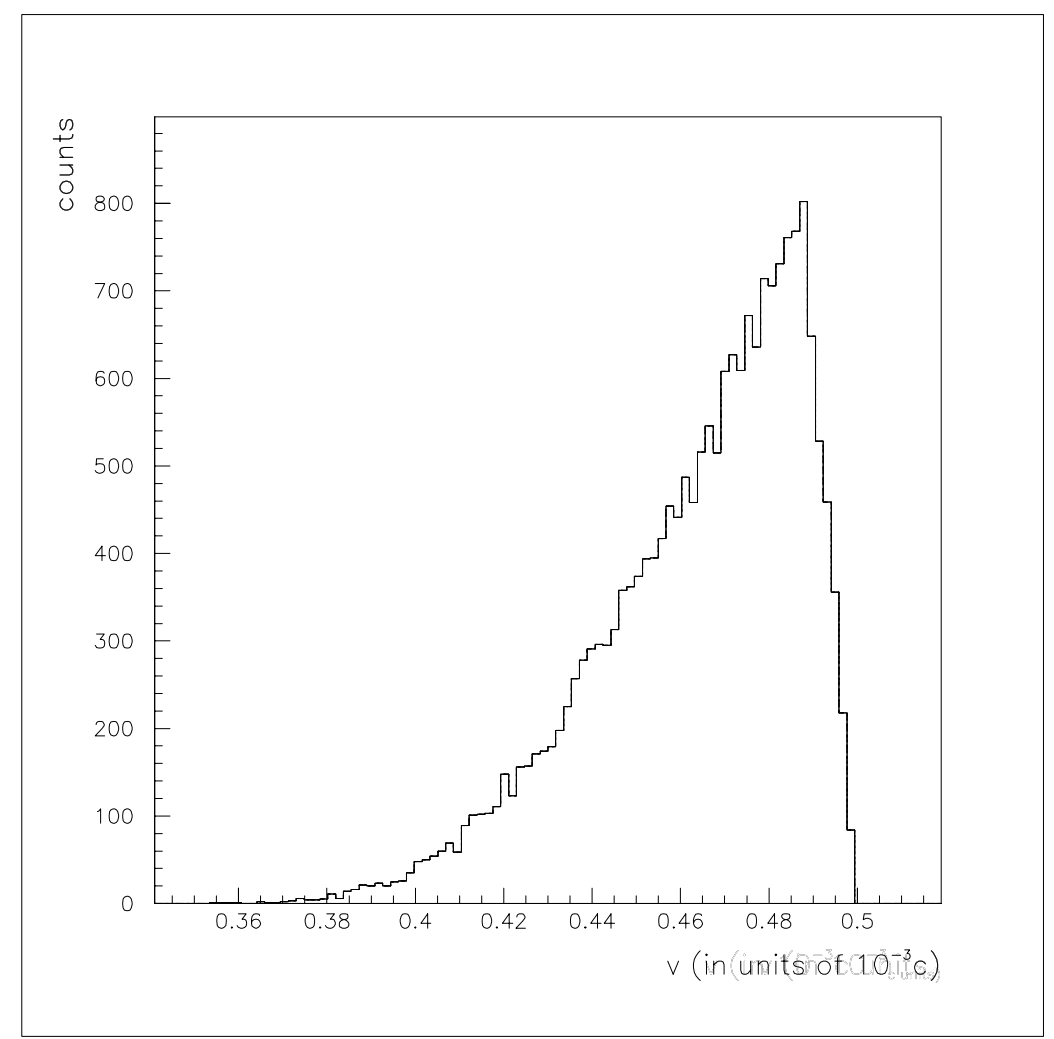

FIGURE 1. Histogram of the velocities at the slit for an ensemble of $20000 \mathrm{H}^{+}$ions. Obtained for $\sigma_{\mathrm{r}}=0.12 \mathrm{~cm}$ and $\sigma_{\mathrm{z}}=12 \mathrm{~cm}$. 
For a more general case of an elliptical beam, the components of the electrical field are different. The ion velocity is no longer radial. In spite of an initial ellipsoidal distribution of ions, the number of ions arriving at the vacuum chamber wall is almost isotropic. The velocity distribution has the same aspect as in Fig. 1. The obtained $\mathrm{v}_{\mathrm{fm}}$ values follow the same logarithmic dependence as a round beam when the radial $\mathrm{rms}$ equals the mean of the $x$ and $y \mathrm{rms}$, that is $\sigma_{\mathrm{r}}=\sigma_{\mathrm{m}} \equiv\left(\sigma_{\mathrm{x}}+\sigma_{\mathrm{y}}\right) / 2$. The distribution width, however, presents azimuthal differences, and can be used to obtain the beam flatness [3]. For the latter propose two detectors measuring simultaneously would be needed, one in the horizontal and another in the vertical plane.

It is important to mention that the $\mathrm{v}_{\mathrm{fm}}$ value has been found to be inversely proportional to the square root of the ion mass. The ion energy remains the same for the same beam conditions, $100-200 \mathrm{eV}$, whereas the arrival velocity decreases as the ion mass increases. This latter effect can also be used as a method of mass spectrometry for low atomic masses. Moreover, the use of $\mathrm{He}$ or $\mathrm{N}_{2}$ gas injected into the beam pipe to increase the local pressure by a factor 10 , has been considered for this detector [3].

Once the ions leave the vacuum chamber through the slit, they are spread by the action of a perpendicular magnetic field, which bends their trajectories. For a uniform constant magnetic field, the bending radius, $\mathrm{R}_{\mathrm{b}}$ is given by:

$$
R_{b}=\frac{M v}{e B}
$$

Where $M$ is the ion mass, $v$ is the ion velocity and $B$ is the magnetic field intensity. For the $\mathrm{H}^{+}$ion, for example, $\mathrm{R}_{\mathrm{b}}[\mathrm{m}]=3.1316 \cdot(v / \mathrm{c}) / \mathrm{B}[\mathrm{T}]$.

The geometric configuration for the detector determines the range of $\mathrm{R}_{b}$ values, of the order of several centimeters. The strength of the magnetic field is therefore a few hundred gauss.

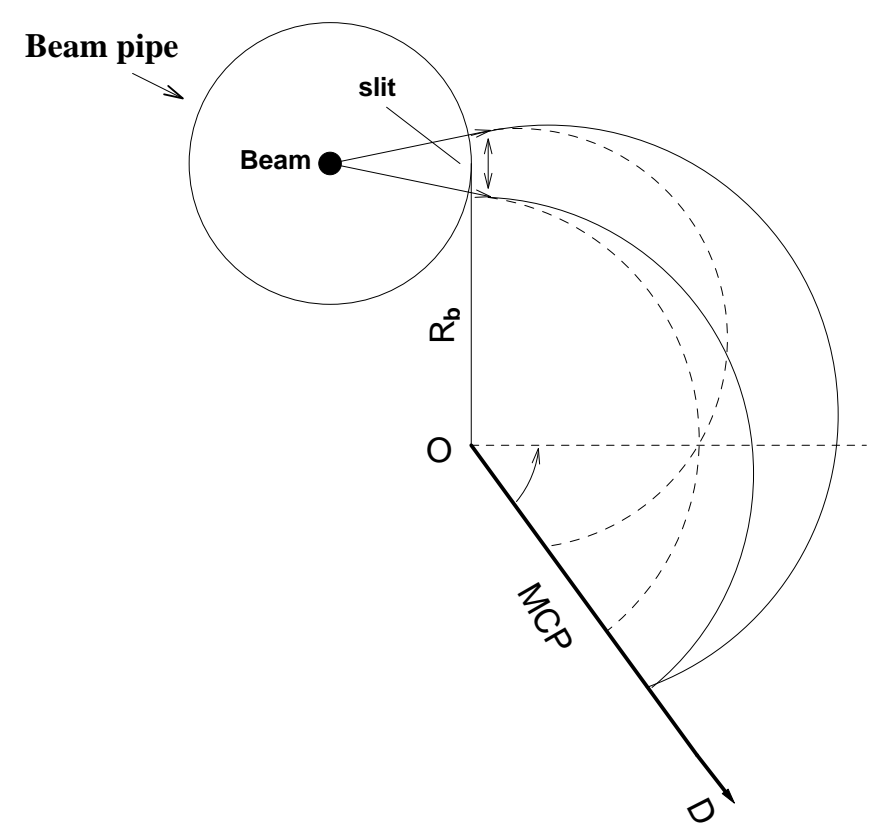

FIGURE 2. Schematic view of the BGIP monitor. The MCP position is determined by the focalization condition for the largest ion trajectory. The variable $D$ specifies the position in the MCP. 
After a while, ions are spread in space as they were in speed, and they can be detected with an ion detector sensitive to their position.

It is worth to state that, the physical width of the slit gives a dispersion of velocity directions for the ions leaving the vacuum chamber. This spread determines the suitable setup of the ion detector to achieve the best resolution. As the beam size is directly determined by the $\mathrm{v}_{\mathrm{fm}}$ value, the goal is to get the different trajectories for this velocity focalized on the detector. A scheme for a general experimental setup is shown in Fig. 2.

From the results obtained in the simulations for the future LHC beam, it has been concluded that this detector can achieve accuracy better than $1 \%$ in the beam dimensions measurement [3]. Moreover, the detector can be used in the whole LHC energy range, from injection energies $(450 \mathrm{GeV})$ to collision energies $(7 \mathrm{TeV})$.

\section{SPS particularities}

To check the performance of this detector for its future use in the LHC beam, it needs to be used in a test beam. This is to be done in the LHC type beams in the SPS ring. The SPS ring is the future injector for the LHC.

We need to analyze the experimental conditions for the SPS tests. The residual gas pressure is of $10^{-8}$ torr, and the bunch intensity $0.7-0.8 \cdot 10^{11}$ protons per bunch. Only $1 / 11$ of the SPS ring is filled with bunches, 84 bunches in total. A detector tank has been installed at the point 41459 of the SPS ring. The slit has an angular aperture of $0.04 \mathrm{rad}$, and a length of $2 \mathrm{~cm}$. Considering these parameters, an ion rate of $2.3 \cdot 10^{5}$ ions/s or $0.04 \mathrm{pA}$ is obtained.

The beta functions at the installation point are quite similar for the horizontal and for the vertical plane, $\beta_{\mathrm{H}}=53 \mathrm{~m}, \beta_{\mathrm{V}}=48 \mathrm{~m}$. The emittance, however is bigger in the horizontal plane, this causes a flatness of the SPS test beam. As a consequence, for the calculations we will take into account the mean value of the beam size, $\sigma_{\mathrm{m}}$.

The energy of the ions will vary between $90 \mathrm{eV}$ and $180 \mathrm{eV}$ for a beam intensity of $0.5-1.0 \cdot 10^{11}$ protons/bunch.

It has to be noticed that the fact of having a filling of 1/11 of the SPS ring produces a change in the velocity spectrum. The number of kicks felt by an ion before leaving the vacuum chamber goes from 20 to 60 , depending mainly on the beam intensity, on the position where the ion is created and on the ion mass. As there is a hole in every SPS turn, the energy spectrum goes down to near zero values. In consequence, the distribution showed in Fig. 1 broadens and the tail is extended in the lowest speed range. The right edge, however, does not change.

\section{DETECTOR PROTOTYPE FOR THE SPS BEAM}

For the detector prototype for the SPS the design must be accommodated to some geometrical parameters. In the SPS ring there is only one beam and the beam pipe dimension is larger, $7.8 \mathrm{~cm}$ radius, than in the future LHC. Beyond the slit, ions are led through a non-magnetic tube, $27.2 \mathrm{~cm}$ long, up to the magnet. In addition, the detector configuration has been dessigned to accomplish focalization for the largest 
velocities, as shown in Fig. 2. Finally, in a realistic case, the magnetic field gradient in the magnet borders has also to be taken into account.

The BGIP prototype built for the SPS is made up of the following main components:

- Ion collector: beam vacuum chamber with a slit allowing the passage of ions and a shielded free drift space tube that guides them into the spectrometer area.

- Dipole magnet with a strength in the range of $0.01 \mathrm{~T}$ to $0.06 \mathrm{~T}(2.5 \mathrm{~A})$.

- Ion position sensor, consisting of a grounded metal grid, a two stage MCP detector at about $-2.2 \mathrm{kV}$ and a phosphor screen at about $7.0 \mathrm{kV}$.

- Optical system. The photon flux emitted by the phosphor screen is collected through a window by a lens of $25 \mathrm{~mm}$ focal length and a 12-bit CCD camera.

- Acquisition system: BEUV application ${ }^{1}[4]$. It allows to load the $2 \mathrm{D}$ camera image in a computer every $30 \mathrm{~s}$ with an integration window of $20-60 \mathrm{~ms}$.

The ion position sensor has been manufactured by Photek Ltd upon specifications. It is placed in a vacuum chamber inside the magnet gap. Fig. 3 represents the SPS prototype, showing the final part of the ion collector, the dipole magnet and the ion position sensor.

To determine the most suitable configuration for the ion position detector set, the ion trajectories have been calculated with an experimental map of the magnetic field. The position of the magnet in relation with the shielding tube has been optimized to have a sharp edge in the magnetic field gradient.

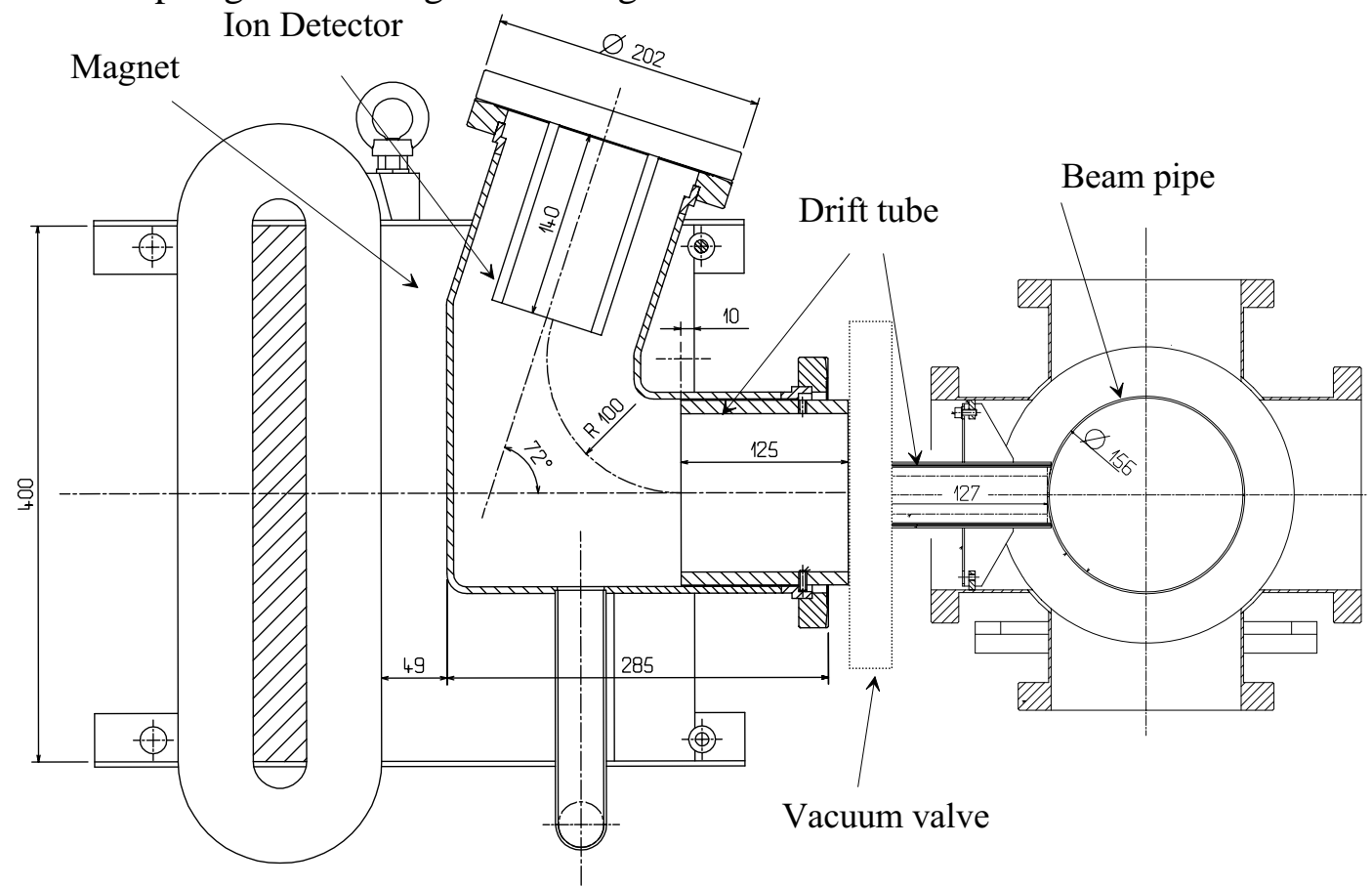

FIGURE 3. Experimental set-up of the BGIP first prototype built for the SPS ring. In the right part, the tank containing the beam pipe and the first section of the drift tube are shown. In the left part are drawn the second section of the drift tube and the ion detector inside its vacuum chamber placed in the magnet gap. The theoretical trajectory of an ion is shown with a perfect curvature radius of $10 \mathrm{~cm}$. Units are given in millimeters.

${ }^{1}$ BEUV: Beam Envelope in the UV. Acquisition system used in the LEP synchrotron light telescopes [4]. 
The MCPs and the phosphor screen have a rectangular shape. Horizontally they cover $65 \mathrm{~mm}$, where the position spectrum is collected. Vertically they cover $21 \mathrm{~mm}$ according to the $20 \mathrm{~mm}$ length of the beam pipe slit. If the measured signal is projected in the horizontal dimension, the obtained distribution is similar to that shown for the velocities in Fig. 1. Vertically we have a flat distribution.

The CCD and its associated optics give a resolution of $200.3 \mu \mathrm{m} / \mathrm{pixel}$.

The applied voltages to the MCPs and the phosphor screen allow to control the gain of the detection system. The MCPs are a pair of micro-channel plates in chevron configuration. The assembly has been tested to have an efficiency of $60 \%$ for hitting ion energies of more than $2 \mathrm{keV}$. This energy is reached by means of the grounded grid and the input voltage in the first $\mathrm{MCP}, \mathrm{V}_{1 \mathrm{i}} \leq-2.2 \mathrm{kV}$. The voltage applied to the phosphor screen, $\mathrm{V}_{\mathrm{ph}}$, ranges between 4.0 and $7.0 \mathrm{kV}$.

\section{TESTS RESULTS}

The BGIP monitor is designed to obtain the beam size from the magnetic field intensity. Once this detector has been checked, the position of the ion distribution on the MCP is fixed. The velocity distribution is translated in a distribution with a maximum position value, $\mathrm{D}_{\mathrm{m}}$ which corresponds to the maximum velocity value, $\mathrm{v}_{\mathrm{fm}}$. This value $\mathrm{D}_{\mathrm{m}}$ in the detector design is situated at $1 \mathrm{~cm}$ of the MCP border, the rest of the position distribution is extended to the other side. The magnetic field, $\mathrm{B}$, is swept until the distribution edge fits the $\mathrm{D}_{\mathrm{m}}$ point. The actual beam size is derived from the strength of this magnetic field.

In the performed tests, we have cross-checked the detector sweeping the field for a fixed beam size. The beam size is obtained from the nearest wire scanner. In this way we can compare the results with the simulations. The mechanism for the detector is not designed to measure $\sigma_{\mathrm{r}}$ (or $\sigma_{\mathrm{m}}$ in this particular case) in this way. The change of $\mathrm{B}$ for the same beam conditions, shifts the $\mathrm{D}_{\mathrm{m}}$ value either to the right or to the left of the focalized point. The focalization gets worse, but it is still good enough to resolve the distribution edge.

\section{Beam Conditions}

Tests were performed at the end of the 1999 SPS run. In that period the LHC type beam available was the Machine Development segment in parallel with physics (lead ion supercycle). The segment length is of $700 \mathrm{~ms}$ without no ramping, having the protons injected at $26 \mathrm{GeV}$.

In the various tests performed, good results were obtained when operation team provided stable, high intensity proton beams. With these conditions, a signal was observed for a magnetic field ranging from 100 to 250 gauss.

We had a batch of 84 bunches, 25 ns spacing, with an intensity of about $70-80 \%$ of the LHC nominal intensity. The intensity measurement values have an error of less than $10 \%$. The emittance values measured in the wire scanner show a blow up along the batch. We have taken the mean values in the first 50 bunches, as they are the first 
bunches which have a major influence in $\mathrm{v}_{\mathrm{fm}}$. The so obtained emittance has a variation of $8 \%$.

\section{Measurements}

The signal obtained with the CCD camera was like expected. We could observe an almost flat distribution in the vertical plane, and a peaked distribution in the horizontal plane. As the magnetic field was swept, the whole image swept at the same time. A design error in the ion detector caused an over-lighting of the phosphor screen borders. That effect prevented us to cover the whole range in the CCD camera.

The simulations performed with the provided beam conditions fit very well the experiments within the error given by the beam parameters inherent variations. We could see that the observed ion was $\mathrm{H}_{2}^{+}$. We were not able to see the ion $\mathrm{H}^{+}$, which should have been observed at lower fields. The magnetic field is proportional to the square root of the mass for the same beam conditions (velocity is inversely proportional to the square root of the mass).

The reason why we do not observe the velocity spectrum of the $\mathrm{H}^{+}$ion could be due to the effect of bunch filling in the SPS. The tail of the $\mathrm{H}_{2}{ }^{+}$distribution up to very low velocities could hide the $\mathrm{H}^{+}$spectrum. In a continuous bunched beam, we have a narrow velocity spectrum that allows determining different species of ions.

An example of the projection obtained experimentally together with the theoretical prediction is shown in Fig. 4. We can see how well the simulations fit the signal, in the expected $D_{m}$ value as well as in the distribution shape. The magnetic field in this case is 174.3 gauss and the obtained $\mathrm{D}_{\mathrm{m}}=11.9 \mathrm{~cm}$ for the experimental data and $\mathrm{D}_{\mathrm{m}}=12.1 \mathrm{~cm}$ for the simulated data.

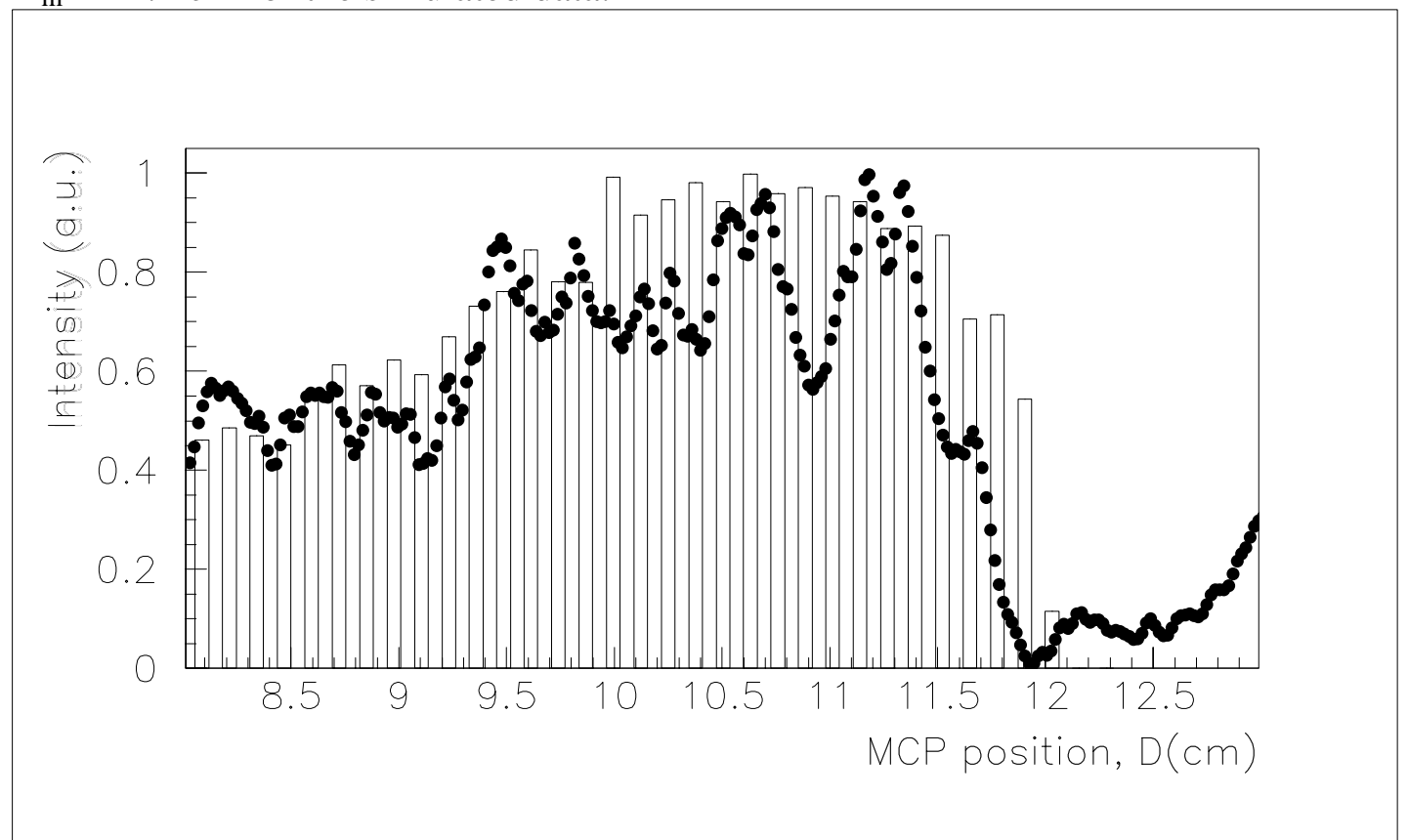

FIGURE 4. Normalized horizontal projection of the signal obtained for the $\mathrm{H}_{2}^{+}$ion at 174.3 gauss (filled circles). $\mathrm{Np}=(0.71 \pm 0.06) \cdot 10^{11} . \sigma_{\mathrm{m}}=4.6 \pm 0.5 \mathrm{~mm}$. The histogram shows the theoretical simulation. 


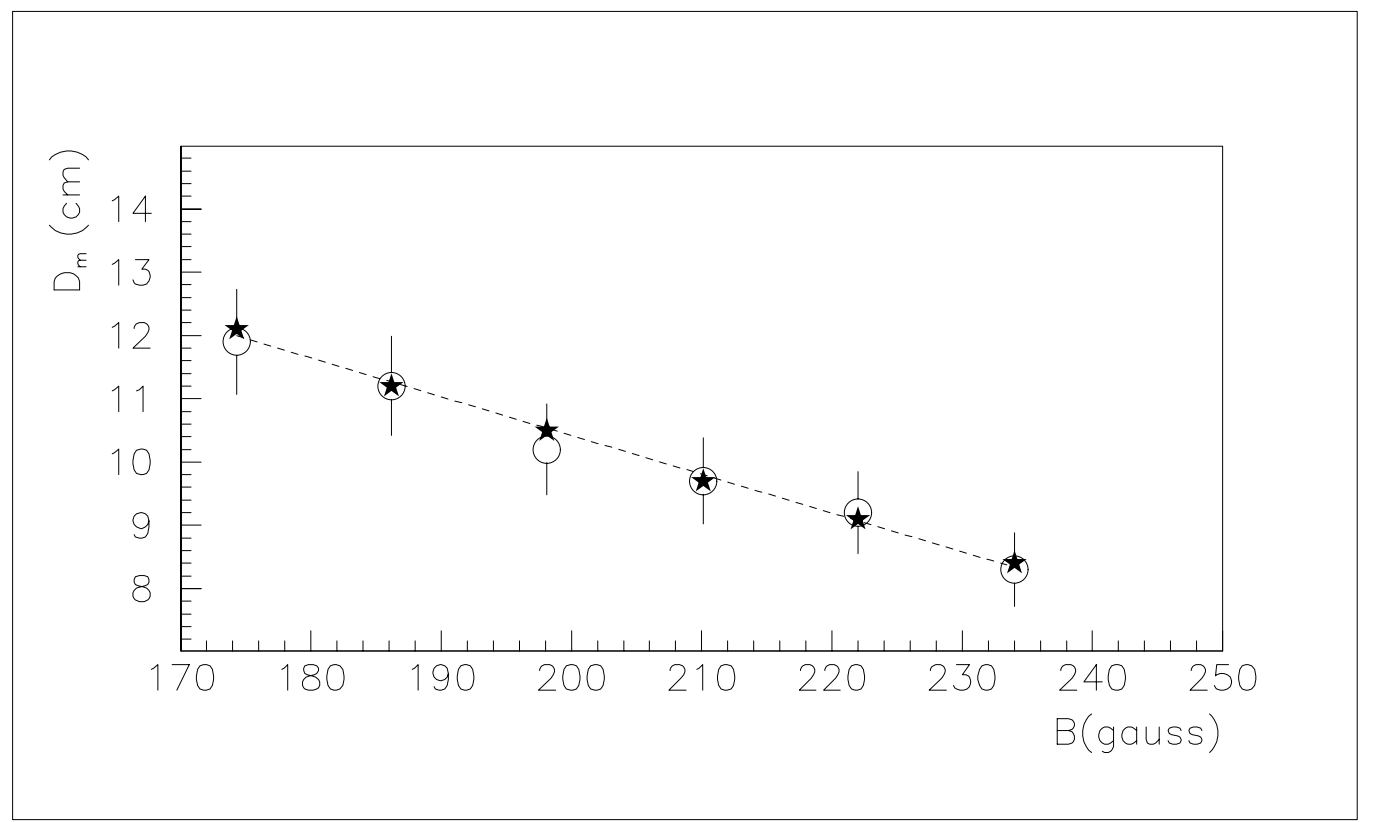

FIGURE 5. Evolution of obtained $D_{m}$ values with magnetic field. Experimental data in open circles with the error bars and theoretical prediction in filled stars. Beam parameters: $\mathrm{Np}=(0.71 \pm 0.06) \cdot 10^{11}$ and $\sigma_{\mathrm{m}}=4.6 \pm 0.5 \mathrm{~mm}$.

The experimental and theoretical values for $\mathrm{D}_{\mathrm{m}}$ for several magnetic field values are plotter in Fig. 5. The error bars plotted for the experimental values come from the beam parameters incertitude. There is a good agreement between both values.

\section{Future tests}

The results obtained from the first test, previously summarized, represent a success for the BGIP monitor. The measuring conditions and the beam parameters are going to be improved for the next SPS 2000 run. The ion position detector has been improved by Photek Ltd to correct the border effect and to increase the resolution by allowing the application of an accelerating voltage between the MCPs. In addition, a Hall probe has been installed to get the B values with a $0.1 \%-0.2 \%$ accuracy.

The acquisition system is also going to be improved by having the possibility of measuring distributions at a high rate in projection mode. Thus, the resolution of the detector will be checked performing several measurements in the same beam cycle.

As for the beam conditions, it is foreseen to have an injection of three batches of 84 bunches each and a ramping up to $450 \mathrm{GeV}$. This would shrink the distribution in the MCP and would allow measuring a range of beam dimensions at the LHC injection energy. Moreover, it is expected to have a better stability in the emittance and in the intensity of the bunches. The precision in the intensity measurement will be of $1 \%$. The intensity error is the major limitation for the precision of this detector, the precision in the $\sigma_{\mathrm{m}}$ value will be $3 \%$ or $4 \%$. In the future LHC this problem is overcome as the intensity measurement is expected to have a great precision. 


\section{SUMMARY}

The BGIP monitor has been proved to be able to measure the beam size of a LHC type beam. Beam size is obtained from the maximum velocity of the residual gas energy spectrum. This is measured by determining the minimum bending by an applied perpendicular magnetic field.

The first detector prototype has been built for the LHC injector, the SPS ring. It is mainly made up of a dipole magnet as a spectrometer combined with an Ion position detector (MCPs). The first tests have been successful, showing a correct performance of the BGIP monitor and a good agreement with theoretical simulations.

New more complete tests are foreseen for a near future, the next SPS run all along this year 2000 .

\section{REFERENCES}

1. Buon J. et al., NIM A306, 93-111 (1991).

2. Puzo P. et al., NIM A425, 415-430 (1999).

3. Arauzo A. et al., LHC Project Note 198 (1999).

4. Bovet C. et al., CERN SL/91-25 (1991).

\section{ACKNOLEDGMENTS}

We would like to thank all the people who have been collaborating in this project and have made possible the final realization of the BGIP monitor. J. Buon and P. Puzo for the support in the theoretical calculations and detector design, R. Jung for his interest and general support, R. Perret, J. Camas, R. Colchester and M. Sillanolli for the help provided in the design and the practical realization and installation, Gijs de Rijk for the magnetic measurements, J.J. Gras for the software support, and finally the vacuum group LHC-VAC for all the services provided. 